Р.И. Овчинников ${ }^{1 凶}$, ORCID: 0000-0001-8219-5216, e-mail: riododc@rambler.ru, r_ovchinnikov@oparina4.ru

С.И. Гамидов ${ }^{1,2}$, ORCID: 0000-0002-9128-2714, e-mail: safargamidov@yandex.ru

A.Ю. Попова ${ }^{1}$, ORCID: 0000-0002-3038-4792, e-mail: Alina-dock@ya.ru

C.X. Ижбаев ${ }^{1}$, ORCID: 0000-0001-8117-3197, e-mail: izhbaev-sergei@mail.ru

${ }^{1}$ Национальный медицинский исследовательский центр акушерства, гинекологии и перинатологии имени академика В.И. Кулакова; 117997, Россия, Москва, ул. Академика Опарина, д. 4

2 Первый Московский государственный медицинский университет имени И.М. Сеченова (Сеченовский Университет); 119991, Россия, Москва, ул. Трубецкая, д. 8, стр. 2

\title{
Резюме
}

Установлено, что мужчины болеют коронавирусом в 1,5 раза чаще, чем женщины, но в то же время 95\% из них переносят инфекцию в легкой форме.

Существует вопросы, которые требуют научного объяснения: почему мужчины болеют чаще и более восприимчивы, чем женщины, проходит ли вирус через гемато-тестикулярный барьер, проникает ли он в семенную жидкость, если ли влияние SARS-CoV-2 на выработку андрогенов, поражается ли яичко и каковы последствия этого поражения, каково непосредственное влияние вируса и связанных с ним проблем (таких как социальные проблемы, изоляция, карантин, психологические проблемы, малоподвижный образ жизни и пр.), прежде всего на сексуальную функцию, сексуальное поведение и репродукцию в целом?

В обзорной статье рассмотрена ситуация, связанная с пандемией коронавируса SARS-CoV-2 в 2020 г. и ее влиянием на мужское здоровье. Приводятся данные по эпидемиологии, аспектам патогенеза, особенностям новой коронавирусной инфекции у мужчин. Озвучены рекомендации международных обществ по репродукции (ASRM, ESHRE, PAPU) по проведению программ вспомогательных репродуктивных технологий (ВРТ) в эпоху пандемии. Представлены разные точки зрения на влияние тестостерона на заболеваемость мужчин. Показана важность оксидативного стресса сперматозоидов и антиоксидантной терапии мужского бесплодия. Изложены задачи международного проекта по изучению влияния коронавирусной инфекции SARS-CoV-2 и ее последствий на здоровье мужчин. Опубликовано приглашение к сотрудничеству с отечественными и зарубежными специалистами в области урологии и андрологии на базе ФГБУ «Национальный медицинский исследовательский центр акушерства, гинекологии и перинатологии имени академика В.И. Кулакова» Министерства здравоохранения Российской Федерации.

Ключевые слова: мужское бесплодие, пандемия, SARS-CoV-2, COVID-19, тестостерон, оксидативный стресс сперматозоидов, антиоксидантная терапия

Для цитирования: Овчинников Р.И., Гамидов С.И., Попова А.Ю., Ижбаев С.Х. Мужское бесплодие: до и после эпохи коронавируса SARS-CoV-2. Медищинский совет. 2020;(13):179-187. doi: 10.21518/2079-701X-2020-13-179-187.

Конфликт интересов: авторы заявляют об отсутствии конфликта интересов.

\section{Male infertility: before and after the era of SARS-CoV-2}

Ruslan I. Ovchinnikov ${ }^{1}$, ORCID: 0000-0001-8219-5216, e-mail: riododc@rambler.ru, r_ovchinnikov@oparina4.ru

Safail I.Gamidov1,2, ORCID: 0000-0002-9128-2714, e-mail: safargamidov@yandex.ru

Alina Yu.Popova1 ${ }^{1}$ ORCID: 0000-0002-3038-4792, e-mail: Alina-dock@ya.ru

Sergey Kh. Izhbaev ${ }^{1}$, ORCID: 0000-0001-8117-3197, e-mail: izhbaev-sergei@mail.ru

${ }^{1}$ National Medical Research Center of Obstetrics, Gynecology and Perinatology named after Academician V.I. Kulakov; 4, Oparin St., Moscow, 117997, Russia

2 I.M. Sechenov First Moscow State Medical University (Sechenov University); 8, Bldg. 2, Trubetskaya St., Moscow, 119991, Russia

\section{Abstract}

It has been found that men are infected with the coronavirus 1.5 times more often than women, but at the same time $95 \%$ of them have mild infection.

There are questions that require a scientific explanation: why are men infected more often and more susceptible than women, does the virus cross the blood-testicular barrier, can it be found in the seminal fluid, does SARS-CoV-2 have an effect on the production of androgens, is the testicle affected and what are the consequences of this damage, what is the direct effect of a virus and virus-associated problems (such as social problems, isolation, quarantine, psychological problems, sedentary lifestyle, etc.) primarily on sexual function, sexual behaviour and reproduction in general?

The review article examines the SARS-CoV-2 pandemic-associated situation in 2020 and its effect on the men's health. The article provides data on epidemiology, aspects of pathogenesis, features of new coronavirus infection in men. The authors announced the 
international reproduction associations (ASRM, ESHRE, RAHR) recommendations for the implementation of assisted reproductive technology (ART) programs in the era of the pandemic. Different points of view on the effect of testosterone on the disease incidence in men are presented. The importance of sperm oxidative stress and antioxidant therapy for male infertility has been shown. The tasks of an international project for the study of SARS-CoV-2 coronavirus infection and its consequences on men's health are outlined. An invitation to collaboration with domestic and foreign specialists in the field of urology and andrology under the aegis of the National Medical Research Center of Obstetrics, Gynecology and Perinatology named after Academician V.I. Kulakov of the Ministry of Health of the Russian Federation has been published.

Keywords: male infertility, pandemic, SARS-CoV-2, COVID-19, testosterone, sperm oxidative stress, antioxidant therapy

For citation: Ovchinnikov R.I., Gamidov S.I., Popova A.Yu., Izhbaev S.Kh. Male infertility: before and after the era of SARS-CoV-2. Meditsinskiy sovet = Medical Council. 2020;(13):179-187. (In Russ.) doi: 10.21518/2079-701X-2020-13-179-187.

Conflict of interest: the authors declare no conflict of interest.

\section{ВВЕДЕНИЕ}

Эта статья не совсем обычная, поскольку она посвящена не отдельным вопросам андрологии, мужского бесплодия, гипогонадизма или сексуальной дисфункции, а создавшейся на сегодняшний момент ситуации, связанной с наличием пандемии коронавируса.

Как вы знаете, с декабря 2019 г. стали появляться первые данные о наличии больных новой коронавирусной инфекцией (SARS-CoV-2) из провинции Ухань (KHP).

В этой работе хотелось бы коснуться не столько научных и теоретических основ изменений, которые провоцирует вирус, сколько прикладных вопросов нашей специальности - урологии, касающихся каждого пациента и практикующего врача-уролога и андролога.

Конечно же, пандемия тяжелого острого респираторного синдрома, вызванного коронавирусом SARS-CoV-2, стала величайшим кризисом в новом веке в области здравоохранения, а также политики, экономики и социальной сферы. При этом до конца непонятно, каковы будут ее последствия. Однако уже сейчас определяются некоторые особенности, которые мы можем обсуждать.

\section{НЕРЕШЕННЫЕ ВОПРОСЫ}

Считается, что решение большинства проблем, связанных с мужской репродуктивной системой, в сложившейся ситуации может быть отложено в связи с тем, что это не является приоритетом для систем здравоохранения. Но что мы знаем об андрологических последствиях влияния SARS-CoV-2 [1]?

На сегодняшний день установлено, что мужчины болеют коронавирусом в 1,5 раза чаще, чем женщины, но в то же время 95\% из них переносят инфекцию в легкой форме.

Это позволяет надеяться на не такую высокую смертность, как при атипичной пневмонии (SARS), ближневосточном респираторном синдроме (MERS) или птичьем гриппе (H5N1), свином гриппе (H1N1) и новом птичьем гриппе (H7N9).

Существует ряд вопросов, которые требуют прежде всего научного объяснения: почему мужчины болеют чаще и более восприимчивы, чем женщины, проходит ли вирус через гемато-тестикулярный барьер, проникает ли он в семенную жидкость, если ли влияние SARS-CoV-2 на выработку андрогенов, поражается ли яичко и каковы последствия этого поражения, каково непосредственное влияние вируса и связанных с ним проблем (таких как социальные проблемы, изоляция, карантин, психологические проблемы, малоподвижный образ жизни и пр.), прежде всего на сексуальную функцию, сексуальное поведение и репродукцию в целом?

Имеется ряд вопросов, которые требует рассмотрения и размышления: практически во всех странах прекращены некоторые андрологические и плановые хирургические медицинские вмешательства. В то же время существуют вмешательства, которые нельзя откладывать, например, криоконсервация спермы до лечения онкологического заболевания. Все эти аспекты требуют исследований.

Известно, что пути проникновения коронавируса в клетки лежат через рецепторы ангиотензинпревращающего фермента (АПФ): при этом экспрессия рецепторов АПФ 2 типа была обнаружена в клетках как эндотелия, так и эпителия легочной, сердечно-сосудистой систем, кардиомиоцитах, а также в ткани яичка, клетках Лейдига и клетках семенной плазмы [2].

\section{SARS-CoV-2 И ЯИЧКИ (СПЕРМА)}

Китайские авторы опубликовали информацию о том, что они обнаружили коронавирус в эпителиальных клетках яичка и клетках Лейдига, причем в свое время они увидели там и коронавирусы 1 типа [3]. Тогда как Y. Ding et al. столкнулись с прямой инфекцией в других органах мужчины, но не в яичках [4]. Данные, касающиеся этого аспекта распространения вируса SARS-CoV, не являются последовательными и согласованными [2].

Так, в недавнем исследовании C. Song et al. собрали 12 образцов спермы от выживших пациентов с COVID-19, а также взяли биопсию яичка от умершего пациента с COVID-19: в образцах спермы и при биопсии яичка в тканях не было обнаружено PHK вируса SARS-CoV-2. Эти результаты могут указывать на то, что вирус даже в острой фазе не способен напрямую заразить яички или половые пути мужчины [5].

В мае этого года были опубликованы результаты когортного исследования, которое было проведено в Шанцю (Китай). Авторы отобрали всех мужчин старше 
15 лет с подтвержденным диагнозом COVID-19 и попросили их предоставить образец спермы для тестирования SARS-CoV-2. Среди 50 идентифицированных пациентов 12 не смогли предоставить образец из-за эректильной дисфункции. В общей сложности 38 пациентов были включены в исследование для тестирования спермы: оно было выполнено методом ПЦР в реальном времени. 23 (60,5\%) участника достигли клинического выздоровления, а 15 (39,5\%) находились в острой стадии инфекции. Было выявлено, что 6 (15,8\%) участников имели положительные результаты по SARS-CoV-2: в т. ч. 4 из 15 (26,7\%) пациентов, которые находились в острой стадии инфекции, и 2 из 23 (8,7\%), у которых было полное выздоровление (обнаружен коронавирус даже при наличии отрицательных анализов ПЦР из зева).

Однако при этом не было обнаружено никакой существенной разницы в группах отрицательного и положительного теста спермы на SARS-CoV-2 по возрасту, истории урогенитального заболевания, дням начала заболевания и дням после госпитализации.

Исследователями были сделаны выводы о том, что вирус SARS-CoV-2 может присутствовать в сперме пациентов с COVID-19 и остается какое-то время в сперме переболевших пациентов [6].

Кроме этого, существуют также данные других исследований о том, что дольше всего коронавирус выделяется с фекалиями. Поэтому по наличию вируса в сточных водах можно судить о том, пройден ли пик инфицирования в конкретном регионе [7].

\section{SARS-COV-2 И ВСПОМОГАТЕЛЬНЫЕ РЕПРОДУКТИВНЫЕ ТЕХНОЛОГИИ (ВРТ)}

Практически одновременно ведущими мировыми обществами по ВРТ - 17 марта 2020 г. Американским обществом по репродуктивной медицине (ASRM), 2 апреля 2020 г. Российской ассоциацией репродукции человека (РАРЧ) и 23 апреля 2020 г. Европейским обществом репродукции человека и эмбриологии (ESHRE) было принято решение о приостановлении проведения процедур BPТ на период пандемии. Это было сделано в связи с тем, что влияние SARS-CoV-2 на гаметы, эмбрионы и ранние сроки беременности до конца неизвестно.

Кроме того, клиникам было рекомендовано минимизировать консультации и численность персонала, время приема и нахождения пациентов в клинике, проводить термометрию всех пациентов и разделение потоков, консультации онлайн или по телефону. Предложено отложить циклы ЭКО, если ситуация не связана с онкологическими заболеваниями или хирургическим лечением половых органов. Был отложен перенос эмбрионов во всех начатых циклах (в т. ч. криоперенос). Вопрос с начатыми циклами овуляции решался в индивидуальном порядке. Было введено обязательное консультирование пациентов о неизвестных науке рисках передачи SARS-CoV-2 эмбрионам и гаметам ${ }^{1}$. 1 ASRM: Patient Management and Clinical Recommendations During the Coronavirus (COV-
ID-19) Pandemic As of March 17, 2020. Available at: https://www.asrm.org/globalassets/asrm/ asrm-content/news-and-publications/covid-19/covidtaskforce.pdf.
При этом не учитывались и не лечились пациенты, несмотря на старший репродуктивный возраст, низкий яичниковый резерв и низкие показатели антимюллерова гормона.

Таким образом, можно сделать вывод, что прекращение на длительный срок оказания медицинской помощи пациентам с бесплодием из-за пандемии SARS-CoV-2 будет иметь пагубные последствия.

Если мы будем сокращать объем андрологической помощи, то не сможем помочь большой группе пациентов. При этом количество нерожденных детей, например, с помощью программ ВРТ, может теоретически быть гораздо больше, чем количество смертей от коронавирусной инфекции. А значит, это будет иметь отрицательные демографические результаты.

Более того, «окно рождаемости» при лечении мужчин с бесплодием может быть временным. Мы можем получить какие-то улучшения, но не добиться наступления беременности и рождения ребенка. Если вовремя не зафиксировать эти положительные изменения (например, показатели спермограммы), можно впоследствии никогда не повторить данный успех в лечении.

Откладывание диагностики и лечения этих мужчин может поставить под угрозу перспективы биологического родительства в принципе. У некоторых категорий пациентов, как и у женщин с низким овариальным резервом, время бежит быстро.

Необходимо продолжать предоставлять андрологические услуги в продуманном порядке и в безопасных условиях, хотя в большинстве стран услуги по лечению бесплодия считаются низкоприоритетными [8].

Прежде всего, говоря о приоритетности, мы должны на первое место поставить пациентов с наличием онкологических заболеваний, т. к. после начала агрессивной терапии основного заболевания - химиолучевой терапии мы можем получить необратимые повреждения сперматогенеза, если не проведем предварительное сохранение сперматозоидов, криоконсервацию спермы.

По результатам исследований, до 30\% выживших мужчин теряют потенциал фертильности после противоопухолевой терапии. Химиотерапия, лучевая терапия и радикальные хирургические процедуры могут необратимо нарушить сперматогенез и эякуляцию, да и само заболевание (к примеру, рак яичка) может также повлиять на фертильность. Поэтому в настоящее время единственным надежным методом сохранения фертильности у мужчин репродуктивного возраста с онкозаболеваниями является криоконсервация сперматозоидов, при этом сперма должна быть сохранена до начала гонадотоксической терапии. Расходы, связанные с криоконсервацией сперматозоидов, относительно невелики, поэтому большинство пациентов бывают удовлетворены принятием такого решения [9].

Пока разрешена только криоконсервация сперматозоидов у мужчин с онкологическими заболеваниями. При этом было предложено внести изменения в существующий порядок и разрешить криоконсервацию паци- 
ентам, проходящим медицинское или хирургическое лечение для улучшения количества или качества спермы, мужчинам репродуктивного возраста, страдающим воспалительными заболеваниями, которые находятся в стадии ремиссии, а также мужчинам репродуктивного возраста с системными аутоиммунными заболеваниями, которые собираются начать лечение гонадотоксическими препаратами [8].

\section{ТАКТИКА У БОЛЬНЫХ АЗООСПЕРМИЕЙ ПРИ ВАРИКОЦЕЛЕ ИЛИ ПОЗДНЕМ ОТЛОЖЕННОМ ОТЦОВСТВЕ}

Как известно, сперматогонии, сперматоциты и ранние сперматиды уязвимы к тепловому и оксидативному стресу, связанному с варикоцеле [10]. Существует множество метаанализов, где было показано, что после устранения варикоцеле у 40\% пациентов возникают шансы на появление сперматозоидов в эякуляте, пригодных для ИКСИ или криоконсервации [11]. Однако эти пациенты должны подвергаться мониторингу в связи с риском рецидива азооспермии [12].

После появления сперматозоидов через некоторое время имеется риск их повторного исчезновения по разным причинам, в т. ч. из-за рецидива варикоцеле. Если сейчас таким пациентам не оказывается помощь, а их, конечно, тоже надо направлять на криоконсервацию сперматозоидов для подстраховки на случай рецидива азооспермии, то потом может быть поздно.

Что касается позднего отложенного отцовства, то таким мужчинам рекомендуется создать банк собственных сперматозоидов в молодом возрасте для планирования репродукции в дальнейшем $[13,14]$.

Еще до «коронавирусной эпохи» в исследованиях было показано, что негативное влияние на фертильность может оказать повреждение ДНК сперматозоидов, причиной которого в том числе часто становится поздний отцовский возраст. Но если у женщин с возрастом уменьшается целостность ооцитов и овариальный резерв, то у мужчин существует способность поддерживать определенный уровень фертильности в течение всей жизни, однако эта функция постепенно снижается. При этом происходит снижение уровня половых гормонов, ухудшение сексуальной функции и показателей сперматогенеза, возрастает вероятность неблагоприятных исходов беременностей, происходят мутации спермы de novo (растут на 4\% в год), а также хромосомные анеуплоидии и врожденные дефекты и заболевания у потомства [15].

С возрастом у мужчины происходит сокращение объема спермы, ухудшается подвижность и морфология сперматозоидов. И хотя их концентрация достоверно не меняется, уменьшается общее количество. Причиной этих возрастных изменений становятся, прежде всего, накопленные сопутствующие заболевания: сосудистая недостаточность (диабет, артериальная гипертензия), хронические инфекции (простатит и др.), ожирение, гормональная недостаточность, гонадотоксины [16].
Также у мужчин увеличивается количество сперматозоидов с ядерными большими вакуолями, значительно возрастает степень фрагментации ДНК, что может приводить к репродуктивным потерям. Поэтому возраст не только матери, но и отца влияет на репродуктивные способности пары [17].

При этом необходимо учесть, что пожилой возраст является фактором риска осложнений SARS-CoV-2, а тяжелую форму SARS-CoV-2 можно лечить неспецифическими противовирусными препаратами с возможными гонадотоксическими эффектами [18].

Поэтому пациенты, планирующие детей в позднем возрасте, обеспокоены опасностью инфицирования SARS-CoV-2 и тем, как эта инфекция способна повлиять на фертильность.

Однако и для молодых пациентов SARS-CoV-2 несет значительные риски, связанные с комплексным влиянием на фертильность. Так, в исследовании с участием 300 пациентов 18-75 лет с SARS-CoV-2 было наглядно продемонстрировано, что немаловажным механизмом, возникающим при гипоксемической дыхательной недостаточности, связанной с SARS-CoV-2, является воспалительная реакция и окислительный стресс. Четкой корреляции между возрастом испытуемых и выраженностью оксидативных повреждений при этом не прослеживалось².

Кроме окидативного стресса, вызванного вирусами и цитокиновыми реакциями, характерными для SARSCoV-2, негативное влияние на фертильность оказывают гипертермия [19] и противовирусные препараты, обладающие генотоксичностью [20, 21].

Вышеизложенное определяет потенциальную стратегию лечения и реабилитации, включающую антиоксидантную терапию: дополнительное использование антиоксидантов и витаминных комплексов может модулировать иммунный ответ хозяина и смягчать «цитокиновый шторм».

\section{БОРЬБА С ОКСИДАТИВНЫМ СТРЕССОМ}

Хорошо известно, что причиной многих проблем с фертильностью является оксидативный стресс - широко распространенное патологическое состояние, признаки которого можно обнаружить у большинства мужчин с бесплодием. Это повреждение активными формами кислорода клеток сперматогенеза (прежде всего - сперматозоидов), при этом источниками оксидативного стресса могут быть и сами сперматозоиды, и лейкоциты спермы.

Причины оксидативного стресса различны. Это и сопутствующие заболевания, такие как сахарный диабет, сердечно-сосудистые и системные, а также онкологические заболевания, и влияние неблагоприятных факторов образа жизни, курение, ожирение, алкоголизм, употребление наркотических и психотропных веществ. Все это

2 https://clinicaltrials.gov/ct2/show/NCT04466657?term=Antioxidant \&cond=covid \&dra $\mathrm{W}=2$ \& rank $=2$. 
может приводить к значительному снижению репродуктивного потенциала мужчины.

Почему же сперматозоиды так уязвимы перед оксидативным стрессом? Сперматозоид - это клетка, представляющая собой ядро, несущее генетический материал, снабженное жгутиком, который обеспечивает подвижность. Мембраны сперматозоидов содержат большое количество полиненасыщенных жирных кислот, и когда оксидативный стресс повреждает мембрану, снижается их подвижность. Сперматозоиды почти не имеют собственной антиоксидантной системы в цитоплазме, поэтому их ДНК беззащитна перед прямым воздействием активных форм кислорода. Но в семенной плазме (жидкости, окружающей сперматозоиды) содержатся защитные факторы. Поэтому с активными формами кислорода и свободными радикалами можно бороться, применяя антиоксидантную терапию.

В одной из работ французских авторов в 2014 г. изложена стратегия по оптимизации фертильности: предлагается оптимизировать образ жизни, а также устранить варикоцеле и/или применять антиоксидантную терапию [15]. Она включена в рекомендации Европейской ассоциации урологов года как терапия, улучшающая результаты ВРТ. А по данным обзора Cochrane 2014 г., употребление в пищу антиоксидантов и микроэлементов способствует повышению шансов на зачатие у субфертильных пар. При этом в исследованиях указывается на отсутствие увеличения риска репродуктивных потерь [22].

Следует сказать, что антиоксидантная терапия при наличии оксидативного стресса в первую очередь влияет на подвижность и концентрацию сперматозоидов, что повышает у пары шансы на возможность наступления естественной беременности. В то же время она практически не влияет на морфологию сперматозоидов.

В терапии субфертильных пар у андрологов широко используется комбинированный препарат Андродоз на основе биологически активных веществ, витаминов и микроэлементов. Эти компоненты обладают антиоксидантным действием и могут улучшать качество сперматогенеза и сперматозоидов. Андродоз содержит L-аргинин, L-карнитин, L-карнозин, коэнзим Q10, глицирризиновую кислоту, цинк, витамин Е, витамин А и селен в дозах, которые при рекомендованном режиме приема по 4 капсулы в день могут обеспечить 12-80\% от уровня суточного потребления.

Особый интерес представляют результаты лечения комплексом Андродоз мужчин, которым впоследствии с целью преодоления бесплодия применяли ВРТ. Одним из инструментов, направленных на повышение эффективности ВРТ, является тест на связывание с гиалуроновой кислотой (НВА-тест), который позволяет провести отбор зрелых и функционально состоятельных сперматозоидов, готовых к оплодотворению яйцеклетки. Изучение влияния терапии Андродозом на показатели НВА-теста и наступление беременности в протоколах ВРТ было проведено А.Ю. Поповой, Р.И. Овчинниковым и С.И. Гамидовым в 2019 г. [23].
При этом оказалось, что после приема препарата доля пациентов с нормальной концентрацией сперматозоидов в эякуляте увеличилась с 68\% до 76\%, отмечалась положительная динамика подвижности сперматозоидов у пациентов, имевших значения этого показателя до лечения в пределах 15-32\%, и увеличение количества морфологически нормальных форм сперматозоидов. В то же время показатели оценки НВА-теста и частота наступления беременности после первой попытки применения ВРТ у мужчин, получивших лечение, оказались значительно более высокими по сравнению с аналогичными показателями у не получавших Андродоз мужчин в контрольной группе. Это позволило авторам рекомендовать терапию Андродозом для усиления воздействия на функциональную способность сперматозоидов и повышения частоты положительных исходов программ ВРТ.

\section{SARS-COV-2 И TEСТОСТЕPOH}

Одним из главных вопросов, касающихся влияния коронавируса на мужское здоровье, является взаимодействие между SARS-CoV-2 и тестостероном. Поскольку мужчины болеют коронавирусной инфекцией в 1,5 раза чаще, чем женщины, и среди мужчин наблюдается более высокая летальность во всех возрастных группах, эти факты требуют определенных объяснений. Есть наблюдения, где показано, что концентрация тестостерона в плазме снижается как с возрастом, так и при наличии сопутствующих заболеваний, таких как диабет, ожирение и обструктивное апноэ сна [24].

Следует понимать, что SARS-CoV-2 инфицирует альвеолярные эпителиальные клетки легких, используя в качестве входа рецептор АСЕ2. Этот рецептор содержится в клетках Лейдига, что подразумевает возможное участие яичка и тестостерона в патогенезе COVID-19 [25].

Усугубляет ли низкое содержание тестостерона коронавирусную инфекцию? Информация, которая существует на сегодняшний день по этому вопросу, противоречива [26].

Есть данные, что низкий уровень тестостерона приводит к большей частоте случаев заражения и увеличению тяжести заболевания коронавирусной инфекцией, поскольку провоспалительные цитокины играют центральную роль в прогрессировании инфекции COVID-19, а наличие гипогонадизма исходно связано с повышением провоспалительных цитокинов. По мнению авторов данного исследования, снижение активности цитокинов или их рецепторов является одним из вариантов лечения пациентов, поскольку тестостерон способен снижать количество IL-6, факторов некроза опухоли и других провоспалительных цитокинов [27].

Также тестостерон может играть важную роль в каскаде событий, приводящих к прогрессированию инфекции COVID-19 вследствие «цитокинового шторма». А подавление экспрессии ACE2 воспалительными цитокинами может увеличивать смертность от COVID-19 [28].

Но в то же время существуют и противоположные данные: предполагается, что высокий уровень тесто- 
стерона усугубляет течение коронавирусной инфекции. Существует так называемая «тестостероновая теория» развития инфекции, связанная с активацией андрогенных рецепторов транскрипции трансмембранной протеазы серина 2 типа (TMPRSS2), которая способна расщеплять рецепторы к АПФ-2 для усиленного проникновения вируса [29]. Модуляция экспрессии трансмембранной протеазы серина 2 типа тестостероном способствует преобладанию у мужчин инфекции COVID-19 [30].

Трансмембранная протеаза серина 2 типа экспрессируется и на легочном уровне. Таким образом, некоторые авторы предполагают, что использование ее ингибиторов (в т. ч. препаратов для лечения рака предстательной железы (РПЖ)) представляет собой привлекательную цель для лечения COVID-19 [31, 32].

Однако это - только гипотеза, которая может и не подтвердиться, поэтому необходимы дальнейшие исследования. Сегодня ведутся работы, которые оценивают терапевтический и профилактический потенциал лекарств, нацеленных на андрогенную активность: речь идет об ингибиторах андрогенных рецепторов, ингибиторах стероидогенеза, ингибиторах 5-а редуктазы, которые широко используются в терапии пациентов с ДГПЖ [33].

Одно из нашумевших исследований, проведенных в мае этого года в Италии, было посвящено подавлению андрогенов и риску развития инфекции, вызванной вирусом SARS-CoV-2. В нем участвовало более 4 тыс. мужчин с лабораторно подтвержденной инфекцией SARS-CoV-2. По итогам этой работы оказалось, что пациенты с РПЖ, получавшие андроген-депривационную терапию, имели значительно более низкий риск инфицирования по сравнению с пациентами, которые не получали андрогенную блокаду. Разница была обнаружена при сравнении этих пациентов с пациентами с другими типами рака: при этом у больных с онкологическими заболеваниями риск инфицирования SARS-CoV-2 был выше, а пациенты с РПЖ, получавшие андрогендепривационную терапию, оказались частично защищены от инфекции SARS-CoV-2 [34].

Выяснение роли тестостерона в борьбе с инфекцией COVID-19 является насущной проблемой, и этому будут посвящены дальнейшие исследования.

\section{ПPOEKT PROTEGGIMI}

Скоро будет опубликован протокол международного исследования по влиянию SARS-CoV-2 на здоровье мужчин (PROTEGGIMI) [35].
Задачи проекта:

1. Разработка международного (европейского) реестра данных, предоставляющего информацию о демографических, эпидемиологических и патологических/функциональных результатах лабораторно подтвержденного SARS-CoV-2 у мужчин, а также о здоровых контрольных группах того же этнического происхождения и возраста.

2. Оценка гормональной среды у пациентов (в зависимости от возраста, этнической принадлежности, сопутствующих заболеваний, ИМТ, симптомов, терапевтических подходов и результатов), а также в сопоставимых по возрасту здоровых контрольных группах.

3. Изучение геномного профиля мужчин с подтвержденным SARS-CoV-2.

4. Разработка системы культивирования in vitro для изучения воздействия тестостерона на различные ткани, включая человеческий АCE2 в яичке, репродуктивном тракте, эпителиальных и эндотелиальных клетках легких.

5. Разработка модели на животных, которая может повторять различия в показателях общих результатов в зависимости от пола (женщины и мужчины).

6. Исследование изменений качества спермы, связанных с инфекцией SARS-CoV-2, как биологического маркера общего мужского здоровья и мужского репродуктивного потенциала.

ФГБУ «Национальный медицинский исследовательский центр акушерства, гинекологии и перинатологии имени академика В.И. Кулакова» Минздрава России обладает всеми современными возможностями диагностики и лечения любых нарушений, в т. ч. при инфекции SARS-CoV-2. В ФГБУ НМИЦ АГП успешно функционируют 25 лабораторий, выполняющих любые исследования на высочайшем уровне и по всем международным стандартам. Все желающие могут присоединиться к этому проекту. Если у вас будут какие-либо трудности с лабораторной частью, то мы приглашаем к сотрудничеству с лабораториями и отделением андрологии и урологии нашего центра, которому в этом году исполняется 10 лет.

Многие андрологи во всем мире и в нашей стране обеспокоены последствиями инфекции SARS-CoV-2 для своих пациентов. Мы приветствуем обмен мнениями по всем андрологическим аспектам эпидемии COVID-19 и приглашаем всех заинтересованных специалистов для дискуссии, проведения клинических и фундаментальных исследований, а также выработки дальнейшей тактики ведения пациентов в создавшейся ситуации.

Поступила / Received 10.08.2020

Поступила после рецензирования / Revised 31.08.2020 Принята в печать / Accepted 15.09.2020

\section{Список литературы}

1. Simoni M., Hofmann M.C. The COVID-19 pandemics: Shall we expect andrological consequences? A call for contributions to ANDROLOGY. Andrology. 2020;8(3):528-529. doi: 10.1111/andr.12804.

2. Illiano E., Trama F., Costantini E. Could COVID-19 have an impact on male fertility? Andrology. 2020;52(6):e13654. doi: 10.1111/and.13654.

3. Zhao J. M., Zhou G. D., Yan-Ling S., Wang S.S., Yang J.F., Meng E.H. et al. Clinical pathology and pathogenesis of severe acute respiratory syndrome.
Zhonghua Shi Yan He Lin Chuang Bing Du Xue Za Zhi. 2003;17(3):217-221. Available at: https://pubmed.ncbi.nlm.nih.gov/15340561.

4. Ding Y., Zhang Q., Zhongxi H., Huang Z., Che X., Hou J. et al. Organ distribution of severe acute respiratory syndrome (SARS) associated coronavirus (SARS-CoV) in SARS patients: implications for pathogenesis and virus transmission pathways. J Pathology. 2004;203(2):622630. doi: 10.1002/path.1560. 
5. Song C., Wang Ya., Li W., Hu B., Chen G., Xia P. et al. Absence of 2019 novel coronavirus in semen and testes of COVID-19 patients. Biol Reprod. 2020;103(1):4-6. doi: 10.1093/biolre/ioaa050.

6. Li D., Jin M., Bao P., Zhao W., Zhang S. Clinical Characteristics and Results of Semen Tests Among Men With Coronavirus Disease 2019. JAMA Netw Open. 2020;3(5):e208292.2020. doi: 10.1001/jamanetworkopen.2020.8292.

7. Mallapaty S. How sewage could reveal true scale of coronavirus outbreak. Nature. 2020;580(7802):176-177. doi: 10.1038/d41586-02000973-x.

8. Esteves S.C., Lombardo F., Garrido N., Alvarez J., Zini A., Colpi G.M. et al. SARS-CoV-2 pandemic and repercussions for male infertility patients: A proposal for the individualized provision of andrological services. Andrology. 2020;00:1-9. doi: 10.1111/andr.12809.

9. Xu R., Centola G.M., Tanrikut C. Genitourinary cancer patients have worse baseline semen parameters than healthy sperm bankers. Andrology. 2019;7(4):449-453. doi: 10.1111/andr.12602.

10. Agarwal A., Hamada A., Esteves S.C. Insight into oxidative stress in varicocele-associated male infertility: part 1. Nat Rev Urol. 2012;9(12):678-690. doi: 10.1038/nrurol.2012.197.

11. Esteves S.C., Miyaoka R., Roque M., Agarwal A. Outcome of varicocele repair in men with nonobstructive azoospermia: systematic review and meta-analysis. Asian J Androl. 2016;18(2):246-253. doi 10.4103/1008$682 X .169562$.

12. Pasqualotto F.F., Sobreiro B.P., Hallak J., Pasqualotto E.B., Lucon A.M. Induction of spermatogenesis in azoospermic men after varicocelectomy repair: an update. Fertil Steril. 2006;85(3):635-639. doi: 10.1016/j.fertnstert.2005.08.043.

13. Jennings M.O., Owen R.C., Keefe D., Kim E.D. Management and counseling of the male with advanced paternal age. Fertil Steril. 2017;107(2):324328. doi: 10.1016/j.fertnstert.2016.11.018.

14. Bertoncelli Tanaka M., Agarwal A., Esteves S.C. Paternal age and assisted reproductive technology: problem solver or trouble maker? Panminerva Med. 2019;61(2):138-151. doi: 10.23736/S0031-0808.18.03512-7.

15. Belloc S., Hazout A., Zini A., Merviel P., Cabry R., Chahine H. et al. How to overcome male infertility after 40: Influence of paternal age on fertility. Maturitas. 2014;78(1):22-29. doi: 0.1016/j.maturitas.2014.02.011.

16. Zini A., Albert O., Robaire B. Assessing sperm chromatin and DNA damage: clinical importance and development of standards. Andrology. 2014;2(3):322-325. doi: 10.1111/j.2047-2927.2014.00193.x.

17. Moskovtsev S.I., Mullen J.B., Lecker I., Jarvi K., White J., Roberts M., Lo K.C. Frequency and severity of sperm DNA damage in patients with confirmed cases of male infertility of different aetiologies. Reprod Biomed Online. 2010;20(6):759-763. doi: 10.1016/j.rbmo.2010.03.002.

18. Drobnis E.Z., Nangia A.K. Antivirals and Male Reproduction. Adv Exp Med Biol. 2017;1034:163-178. doi: 10.1007/978-3-319-69535-8_11.

19. Виноградов И.В., Живулько А.Р., Королев С.В. Инфекции добавочных половых желез: механизмы влияния на мужскую фертильность. Вестник урологии. 2019;7(4):43-48. doi: 10.21886/2308-6424-2019-7-4-43-48.

20. Жанатаев А.К., Боровская Т.Г., Щербакова В.С., Рудой Б.А., Вычужанина А.В., Григорьева В.А. и др. Оценка генотоксичности препарата Кагоцел. Бюллетень экспериментальной биологии и медицины. 2018;166(12):690669. Режим доступа: http://iramn.ru/journals/bbm/2018/12/1263.

21. Малюченко Н.В., Кошкина Д.О., Коровина А.Н., Герасимова Н.С., Кирпичников М.П., Студитский В.М., Феофанов А.В. Влияние госсипола на структуру нуклеосом. Вестник Московского университета. Серия 16.
Биология. 2020;75(3):170-175. Режим доступа: https://vestnik-bio-msu. elpub.ru/jour/article/view/901/522\#.

22. Showell M.G., Mackenzie-Proctor R., Brown J., Yazdani A., Stankiewicz M.T., Hart R.J. Antioxidants for male subfertility. Cochrane Database Syst Rev. 2014;(12):CD007411. doi: 10.1002/14651858.CD007411.pub3.

23. Попова А.Ю., Овчинников Р.И., Гамидов С.И. Антиоксидантная терапия улучшает показатели НВА-теста у мужчин с бесплодием при подготовке К программам вспомогательных репродуктивных технологий (ЭКО/ ИКСИ). Урология. 2019;(1):90-96. doi: 10.18565/urology.2019.1.90-96.

24. Bhasin S., Brito J.P., Cunningham G.R., Hayes FJ., Hodis H.N., Matsumoto A.M. et al. Testosterone Therapy in Men With Hypogonadism: An Endocrine Society Clinical Practice Guideline. J Clin Endocrinol Metab. 2018;103(5):1715-1744. doi: 10.1210/jc.2018-00229.

25. Douglas G.C., O’Bryan M.K., Hedger M.P., Lee D.K., Yarski M.A., Smith A.I., Lew R.A. The novel angiotensin-converting enzyme (ACE) homolog, ACE2, is selectively expressed by adult Leydig cells of the testis. Endocrinology. 2004;145(10):4703-4711. doi: 10.1210/en.2004-0443.

26. Pozzilli P., Lenzi A. Commentary: Testosterone, a key hormone in the context of COVID-19 pandemic. Metabolism. 2020;108:154252. doi: 10.1016/j. metabol.2020.154252.

27. Mohamad N.V., Wong S.K., Wan Hasan W.N., Jolly J.J., Nur-Farhana M.F., ImaNirwana S., Chin K.Y. The relationship between circulating testosterone and inflammatory cytokines in men. Aging Male. 2019;22(2):129-140. doi: 10.1080/13685538.2018.1482487.

28. Chen J., Jiang Q., Xia X., Liu K., Yu Z., Tao W. et al. Individual variation of the SARS-CoV-2 receptor ACE2 gene expression and regulation. Aging Cell. 2020;19(7):e13168. doi: 10.1111/acel.13168

29. Heurich A., Hofmann-Winkler H., Gierer S., Liepold T., Jahn O., Pöhlmann S. TMPRSS2 and ADAM17 cleave ACE2 differentially and only proteolysis by TMPRSS2 augments entry driven by the severe acute respiratory syndrome coronavirus spike protein. J Virol. 2014;88(2):1293-1307. doi: 10.1128/JVI.02202-13.

30. Stopsack K.H., Mucci L.A., Antonarakis E.S., Nelson P.S., Kantoff P.W. TMPRSS2 and COVID-19: Serendipity or Opportunity for Intervention? Cancer Discov. 2020;10(6):779-782. doi: 10.1158/2159-8290.CD-20-0451.

31. Lukassen S., Chua R.L., Trefzer T., Kahn N.C., Schneider M.A., Muley T. et al. SARS-CoV-2 receptor ACE2 and TMPRSS2 are primarily expressed in bronchial transient secretory cells. EMBO J. 2020;39(10):e105114. doi: 10.15252/embj.20105114.

32. Hoffmann M., Kleine-Weber H., Schroeder S., Krüger N., Herrler T., Erichsen S. et al. SARS-CoV-2 Cell Entry Depends on ACE2 and TMPRSS2 and Is Blocked by a Clinically Proven Protease Inhibitor. Cell. 2020;181(2):271280.e8. doi: 10.1016/j.cell.2020.02.052.

33. Wambier C.G., Goren A. Severe acute respiratory syndrome coronavirus 2 (SARS-CoV-2) infection is likely to be androgen mediated. J Am Acad Dermatol. 2020;83(1):308-309. doi: 10.1016/j.jaad.2020.04.032.

34. Montopoli M., Zumerle S., Vettor R., Rugge M., Zorzi M., Catapano C.V. et al. Androgen-deprivation therapies for prostate cancer and risk of infection by SARS-CoV-2: a population-based study ( $\mathrm{N}=4532)$. Ann Oncol. 2020;31(8):1040-1045. doi: 10.1016/j.annonc.2020.04.479.

35. Salonia A., Corona G., Giwercman A., Maggi M., Minhas S., Nappi R.E. et al. SARS-CoV-2, testosterone and frailty in males (PROTEGGIMI): A multidimensional research project. Andrology. 2020;00:1-4. doi: 10.1111/ andr.12811.

\section{References}

1. Simoni M.,Hofmann M.C. The COVID-19 pandemics: Shall we expect andrological consequences? A call for contributions to ANDROLOGY. Andrology. 2020;8(3):528-529. doi: 10.1111/andr.12804.

2. Illiano E., Trama F., Costantini E. Could COVID-19 have an impact on male fertility? Andrology. 2020;52(6):e13654. doi: 10.1111/and.13654.

3. Zhao J. M., Zhou G. D., Yan-Ling S., Wang S.S., Yang J.F., Meng E.H. et al. Clinical pathology and pathogenesis of severe acute respiratory syndrome. Zhonghua Shi Yan He Lin Chuang Bing Du Xue Za Zhi. 2003;17(3):217-221. Available at: https://pubmed.ncbi.nlm.nih. gov/15340561.

4. Ding Y., Zhang Q., Zhongxi H., Huang Z., Che X., Hou J. et al. Organ distribu tion of severe acute respiratory syndrome (SARS) associated coronavirus (SARS-CoV) in SARS patients: implications for pathogenesis and virus transmission pathways. J Pathology. 2004;203(2):622-630. doi: 10.1002/ path.1560.

5. Song C., Wang Ya., Li W., Hu B., Chen G., Xia P. et al. Absence of 2019 novel coronavirus in semen and testes of COVID-19 patients. Biol Reprod. 2020;103(1):4-6. doi: 10.1093/biolre/ioaa050.
6. Li D., Jin M., Bao P., Zhao W., Zhang S. Clinical Characteristics and Results of Semen Tests Among Men With Coronavirus Disease 2019. JAMA Netw Open. 2020;3(5):e208292.2020. doi: 10.1001/jamanetworkopen.2020.8292.

7. Mallapaty S. How sewage could reveal true scale of coronavirus outbreak. Nature. 2020;580(7802):176-177. doi: 10.1038/d41586-020-00973-x.

8. Esteves S.C., Lombardo F., Garrido N., Alvarez J., Zini A., Colpi G.M. et al. SARS-CoV-2 pandemic and repercussions for male infertility patients: A proposal for the individualized provision of andrological services. Andrology. 2020;00:1-9. doi: 10.1111/andr.12809.

9. Xu R., Centola G.M., Tanrikut C. Genitourinary cancer patients have worse baseline semen parameters than healthy sperm bankers. Andrology. 2019;7(4):449-453. doi: 10.1111/andr.12602.

10. Agarwal A., Hamada A., Esteves S.C. Insight into oxidative stress in varicocele-associated male infertility: part 1. Nat Rev Urol. 2012;9(12):678-690. doi: 10.1038/nrurol.2012.197.

11. Esteves S.C., Miyaoka R., Roque M., Agarwal A. Outcome of varicocele repair in men with nonobstructive azoospermia: systematic review and meta-analysis. Asian J Androl. 2016;18(2):246-253. doi 10.4103/1008-682X.169562. 
12. Pasqualotto F.F., Sobreiro B.P., Hallak J., Pasqualotto E.B., Lucon A.M. Induction of spermatogenesis in azoospermic men after varicocelectomy repair: an update. Fertil Steril. 2006;85(3):635-639. doi: 10.1016/j.fertnstert.2005.08.043.

13. Jennings M.O., Owen R.C., Keefe D., Kim E.D. Management and counseling of the male with advanced paternal age. Fertil Steril. 2017;107(2):324328. doi: 10.1016/j.fertnstert.2016.11.018.

14. Bertoncelli Tanaka M., Agarwal A., Esteves S.C. Paternal age and assisted reproductive technology: problem solver or trouble maker? Panminerva Med. 2019;61(2):138-151. doi: 10.23736/S00310808.18.03512-7.

15. Belloc S., Hazout A., Zini A., Merviel P., Cabry R., Chahine H. et al. How to overcome male infertility after 40: Influence of paternal age on fertility. Maturitas. 2014;78(1):22-29. doi: 0.1016/j.maturitas.2014.02.011.

16. Zini A., Albert O., Robaire B. Assessing sperm chromatin and DNA damage: clinical importance and development of standards. Andrology. 2014;2(3):322-325. doi: 10.1111/j.2047-2927.2014.00193.x.

17. Moskovtsev S.I., Mullen J.B., Lecker I., Jarvi K., White J., Roberts M., Lo K.C. Frequency and severity of sperm DNA damage in patients with confirmed cases of male infertility of different aetiologies. Reprod Biomed Online. 2010;20(6):759-763. doi: 10.1016/j.rbmo.2010.03.002.

18. Drobnis E.Z., Nangia A.K. Antivirals and Male Reproduction. Adv Exp Med Biol. 2017;1034:163-178. doi: 10.1007/978-3-319-69535-8_11.

19. Vinogradov I.V., Zhivulko A.R., Korolev S.V. Male Accessory Glands Infections: Mechanisms of Influence on Male Fertility. Vestnik urologii = Urology Herald. 2019;7(4):43-48. (In Russ.) doi: 10.21886/2308-64242019-7-4-43-48.

20. Zhanataev A.K., Borovskaya T.G., Shcherbakova V.S., Rudoy B.A., Vychuzhanina A.V., Grigoreva V.A. et al. Evaluation of the genotoxicity of the drug Kagocel. Byulleten ehksperimental'noy biologii $i$ meditsiny = Bulletin of Experimental Biology and Medicine. 2018;166(12):690-669. (In Russ.) Available at: http://iramn.ru/journals/bbm/2018/12/1263.

21. Malyuchenko N.V., Koshkina D.O., Korovina A.N., Gerasimova N.S., Kirpichnikov M.P., Studitsky V.M., Feofanov A.V. Effect of gossypol on nucleosome structure. Vestnik Moskovskogo universiteta. Seriya 16. Biologiya = Herald of Moscow University. Series 16. Biology. 2020;75(3):170-175. (In Russ.) Available at: https://vestnik-bio-msu.elpub.ru/jour/article/ view/901/522\#

22. Showell M.G., Mackenzie-Proctor R., Brown J., Yazdani A., Stankiewicz M.T., Hart R.J. Antioxidants for male subfertility. Cochrane Database Syst Rev. 2014;(12):CD007411. doi: 10.1002/14651858.CD007411.pub3.

23. Popova A.Yu., Ovchinnikov R.I., Gamidov S.I. Antioxidant therapy improves the results of HBA-test in infertile men during a preparation for assisted reproductive technology (IVF/ICSI). Urologiia = Urology. 2019;(1):90-96. (In Russ.) doi: 10.18565/urology.2019.1.90-96.
24. Bhasin S., Brito J.P., Cunningham G.R., Hayes F.J., Hodis H.N., Matsumoto A.M. et al. Testosterone Therapy in Men With Hypogonadism: An Endocrine Society Clinical Practice Guideline. J Clin Endocrinol Metab. 2018;103(5):1715-1744. doi: 10.1210/jc.2018-00229.

25. Douglas G.C., O’Bryan M.K., Hedger M.P., Lee D.K., Yarski M.A., Smith A.I., Lew R.A. The novel angiotensin-converting enzyme (ACE) homolog, ACE2, is selectively expressed by adult Leydig cells of the testis. Endocrinology. 2004;145(10):4703-4711. doi: 10.1210/en.2004-0443.

26. Pozzilli P., Lenzi A. Commentary: Testosterone, a key hormone in the context of COVID-19 pandemic. Metabolism. 2020;108:154252. doi: 10.1016/j. metabol.2020.154252.

27. Mohamad N.V., Wong S.K., Wan Hasan W.N., Jolly J.J., Nur-Farhana M.F., ImaNirwana S., Chin K.Y. The relationship between circulating testosterone and inflammatory cytokines in men. Aging Male. 2019;22(2):129-140. doi: 10.1080/13685538.2018.1482487.

28. Chen J., Jiang Q., Xia X., Liu K., Yu Z., Tao W. et al. Individual variation of the SARS-CoV-2 receptor ACE2 gene expression and regulation. Aging Cell. 2020;19(7):e13168. doi: 10.1111/acel.13168.

29. Heurich A., Hofmann-Winkler H., Gierer S., Liepold T., Jahn O., Pöhlmann S. TMPRSS2 and ADAM17 cleave ACE2 differentially and only proteolysis by TMPRSS2 augments entry driven by the severe acute respiratory syndrome coronavirus spike protein. J Virol. 2014;88(2):1293-1307. doi: 10.1128/JVI.02202-13.

30. Stopsack K.H., Mucci L.A., Antonarakis E.S., Nelson P.S., Kantoff P.W. TMPRSS2 and COVID-19: Serendipity or Opportunity for Intervention? Cancer Discov. 2020;10(6):779-782. doi: 10.1158/2159-8290.CD-20-0451.

31. Lukassen S., Chua R.L., Trefzer T., Kahn N.C., Schneider M.A., Muley T. et al. SARS-CoV-2 receptor ACE2 and TMPRSS2 are primarily expressed in bronchial transient secretory cells. EMBO J. 2020;39(10):e105114. doi: 10.15252/embj.20105114.

32. Hoffmann M., Kleine-Weber H., Schroeder S., Krüger N., Herrler T., Erichsen S. et al. SARS-CoV-2 Cell Entry Depends on ACE2 and TMPRSS2 and Is Blocked by a Clinically Proven Protease Inhibitor. Cell. 2020;181(2):271280.e8. doi: 10.1016/j.cell.2020.02.052.

33. Wambier C.G., Goren A. Severe acute respiratory syndrome coronavirus 2 (SARS-CoV-2) infection is likely to be androgen mediated. J Am Acad Dermatol. 2020;83(1):308-309. doi: 10.1016/j.jaad.2020.04.032.

34. Montopoli M., Zumerle S., Vettor R., Rugge M., Zorzi M., Catapano C.V. et al. Androgen-deprivation therapies for prostate cancer and risk of infection by SARS-CoV-2: a population-based study ( $\mathrm{N}=4532)$. Ann Oncol. 2020;31(8):1040-1045. doi: 10.1016/j.annonc.2020.04.479.

35. Salonia A., Corona G., Giwercman A., Maggi M., Minhas S., Nappi R.E. et al. SARS-CoV-2, testosterone and frailty in males (PROTEGGIMI): A multidimensional research project. Andrology. 2020;00:1-4. doi: 10.1111/ andr.12811.

\section{Вклад авторов}

Концепция статьи - Овчинников Р. И., Гамидов С.И.

Написание текста - Овчинников Р.И., Ижбаев С.Х.

Обзор литературы - Овчинников Р.И., Ижбаев С.Х., Попова А.Ю.

\section{Contribution of authors}

Concept of article - Ruslan I. Ovchinnikov, Safail I. Gamidov

Text development - Ruslan I. Ovchinnikov, Sergey Kh. Izhbaev

Literature review - Ruslan I. Ovchinnikov, Sergey Kh. Izhbaev, Alina Yu. Popova

\section{Информация об авторах:}

Овчинников Руслан Игоревич, к.М.н., заведующий по клинической работе отделения андрологии и урологии, Федеральное государственное бюджетное учреждение «Национальный медицинский исследовательский центр акушерства, гинекологии и перинатологии имени академика В.И. Кулакова» Министерства здравоохранения Российской Федерации; 117997, Россия, Москва, ул. Академика Опарина, д. 4; e-mail:riododc@rambler.ru,r_ovchinnikov@oparina4.ru

Гамидов Сафар Исраилович, д.м.н., заведующий отделением андрологии и урологии, Федеральное государственное бюджетное учреждение «Национальный медицинский исследовательский центр акушерства, гинекологии и перинатологии имени академика В.И. Кулакова» Министерства здравоохранения Российской Федерации; 117997, Россия, Москва, ул. Академика Опарина, д. 4; профессор кафедры урологии и оперативной нефрологии, Федеральное государственное автономное образовательное учреждение высшего образования «Первый Московский государственный медицинский университет имени И.М. Сеченова» Министерства здравоохранения Российской Федерации (Сеченовский Университет); 119991, Россия, Москва, ул. Трубецкая, д. 8, стр. 2; e-mail: safargamidov@yandex.ru

Попова Алина Юрьевна, к.м.н., старший научный сотрудник отделения андрологии и урологии, Федеральное государственное бюджетное учреждение «Национальный медицинский исследовательский центр акушерства, гинекологии и перинатологии имени академика B.И. Кулакова» Министерства здравоохранения Российской Федерации; 117997, Россия, Москва, ул. Академика Опарина, д. 4; е-таі: Alina-dock@ya.ru 
Ижбаев Сергей Хасанович, к.м.н., уролог, андролог, врач ультразвуковой диагностики отделения андрологии и урологии, Федеральное государственное бюджетное учреждение «Национальный медицинский исследовательский центр акушерства, гинекологии и перинатологии имени академика В.И. Кулакова» Министерства здравоохранения Российской Федерации; 117997, Россия, Москва, ул. Академика Опарина, д. 4; e-mail: izhbaev-sergei@mail.ru

\section{Information about the authors:}

Ruslan I. Ovchinnikov, Cand. of Sci. (Med.), Head of Clinical Affairs, Department of Andrology and Urology, Federal State Budgetary Institution "National Medical Research Center of Obstetrics, Gynecology and Perinatology named after Academician V.I. Kulakov" of the Ministry of Health of the Russian Federation; 4, Oparin St., Moscow, 117997, Russia; e-mail: riododc@rambler.ru, r_ovchinnikov@oparina4.ru

Safail I. Gamidov, Dr. of Sci. (Med.), Head of Department of Andrology and Urology, Federal State Budgetary Institution "National Medical Research Center of Obstetrics, Gynecology and Perinatology named after Academician V.I. Kulakov" of the Ministry of Health of the Russian Federation; 4, Oparin St., Moscow, 117997, Russia; Professor of Department of Urology and Operative Nephrology, Federal State Autonomous Educational Institution of Higher Education “I.M. Sechenov of the Ministry of Health of the Russian Federation (Sechenov University); 8, Bldg. 2, Trubetskaya St., Moscow, 119991, Russia; e-mail: safargamidov@yandex.ru

Alina Yu. Popova, Cand. of Sci. (Med.), Senior Researcher, Department of Andrology and Urology, Federal State Budgetary Institution "National Medical Research Center of Obstetrics, Gynecology and Perinatology named after Academician V.I. Kulakov" of the Ministry of Health of the Russian Federation; 4, Ostrovityanov St., Moscow, 117997, Russia; e-mail: Alina-dock@ya.ru

Sergey Kh. Izhbaev, Cand. of Sci. (Med.), urologist, andrologist, ultrasound specialist, Department of Andrology and Urology, Federal State Budgetary Institution "National Medical Research Center of Obstetrics, Gynecology and Perinatology named after Academician V.I. Kulakov" of the Ministry of Health of the Russian Federation; 4, Oparin St., Moscow, 117997, Russia; e-mail: izhbaev-sergei@mail.ru 